\title{
BUOYANCY AND CHEMICAL REACTION EFFECTS ON MHD FREE CONVECTIVE SLIP FLOW OF NEWTONIAN AND POLAR FLUID THROUGH POROUS MEDIUM IN THE PRESENCE OF THERMAL RADIATION AND OHMIC HEATING WITH DUFOUR EFFECT
}

\author{
Komal Choudhary, Abhay Kumar Jha, Lakshmi Narayan Mishra* and Vandana
}

\begin{abstract}
The present paper investigates the effects of thermal radiation, joule heating on an unsteady hydro magnetic free convective flow of a viscous electrically conductive Newtonian and polar fluid past a semi-infinite vertical plate embedded in a porous media in the presence of heat absorption, chemical reaction, slip flow and Dufour effect. Analytical perturbation solutions are obtained for the velocity, temperature and concentration fields as well as for the skin friction coefficient, Nusselt number and Sharewood number. The results are presented in graphical forms to study the effects of various parameters.
\end{abstract}

Keywords: Newtonian fluid, Polar Fluid, Ohmic Heating, Slip Flow, Dufour effect.

\section{Introduction}

\section{Nomenclature}

$G_{r}$ - Grashof number for heat transfer

$G_{c}$ - Grashof number for mass transfer

$K_{0}$ - Permeability of the porous medium

M - Magnetic field parameter (Hartmann number)

$P_{r}$ - Prandtl number

$\mathrm{S}$ - Heat source parameter

$S_{c}$ - Schmidt number

$D_{u}$ - Dufour number parameter

F - Radiation parameter

Received May 25, 2017; accepted December 13, 2017

2010 Mathematics Subject Classification. Primary 76Dxx; Secondary 76Mxx, 76Nxx,76Rxx,76Sxx,76Wxx.

${ }^{*}$ Corresponding author. 
Uø - Fluid velocity far away from the plate

$\mathrm{t}$ - Time dependent parameter

$V_{0}$ - Reference velocity

$E_{c}$ - Eckert number

$\gamma$ - chemical reaction parameter

\section{Greek symbols}

$v$ - kinematic viscosity

$\theta$ - Dimensionless temperature

$\Omega$ - Angular velocity component

$\alpha$ - Non-Newtonian parameter

Convective heat and the process of mass transfer take place both by diffusion (the random Brownian motion of individual particles in the fluid) and by advection, in which matter or heat is transported by the larger-scale motion of currents in the fluids. Convection of a heated or cooled vertical plate is one of the fundamental problems in heat and mass transfer. Mixed convection is a phenomenon in which the existing free convection is accompanied by an external flow, and the combined mode of free and forced convection exists. In recent years free convective flow past a vertical plate has been studied by many researchers [1]. Martynenko et al. [2] investigated laminar free convection from a vertical plate. Lee and Yovanovich [3] studied the linearization of natural convection from a vertical plate with arbitrary heat flux distribution. Laminar free convection in water with variable physical properties adjacent to a vertical plate with uniform heat flux has been analyzed by Pantokratoras [4]. In several fields of industries and technology, such as petroleum industries, geothermal engineering, energy conservation and underground disposal of nuclear waste material, transport processes in porous media play a significant role. Numerous studies have been done on the problem of free convective and mass transfer flow of viscous fluid through a porous medium [5, 6]. Cheng and Minkowycz [7] analyzed the free convection about a vertical flat plate embedded in porous media with application to heat transfer.

Further heat and mass transfer in the presence of magnetic field has different applications in many emerging fields due to an electromagnetic field. Magnetohydrodynamics is the study of the magnetic properties of electrically conducting fluid such as magneto fluids. Examples include plasmas, liquid metals and electrolyte. This phenomenon is widely used in metallurgy industry, power generators and cooling of nuclear reactors. Helmy [8] analyzed MHD unsteady free convection flow past a vertical porous plate. The flow of unsteady MHD convective heat transfer past a semi-infinite vertical porous plate moving with variable suction has been studied by Kim [9]. Das et al. [10] analyzed the numerical solution of mass transfer effects on unsteady flow past an accelerated vertical porous plate with suction.

Recently, researchers in the engineering and scientific field have shown great interest in the study of the non-Newtonian fluid due to its importance in industrial processes. Fluids for which the relationship between shear stress and the rate of strain is not linear at a given temperature are said to be non-Newtonian. A polar fluid is a fluid where the constituent molecules have a polarization. It could be a fluid of molecules that have a magnetic spin 
moment. Rheological complex fluids such as blood plasma, chocolate, mustard, mayonnaise, clay coatings, certain oils and greases, paints are some examples of non-Newtonian fluids. Many authors have examined the flow, heat and mass transfer in non-Newtonian fluids of different types, e.g., polar fluids. Micro-polar fluids are those which contain microconstituents that can undergo rotation, the presence of which can affect the hydrodynamics of the flow so that it can be distinctly non-Newtonian. Such types of fluid have many applications, for example, analyzing the behavior of lubricants, cooling of the metallic plate in a bath, extrusion of polymer fluid, etc. The theory of micropolar fluids developed by Eringen [11-13] describes a physical system which does not satisfy the Navier-Stokes equations. The unsteady free convective flow of viscoelastic fluids was studied by Soundalgekar [14]. The free convection boundary layer flow of a micro polar fluid from a vertical flat plate was studied by Rees [15]. El-Amin [16] considered MHD free convection and mass transfer flow in a micropolar fluid over a stationary vertical plate with constant suction. Kim [17] analyzed the heat and mass transfer in MHD micropolar flow over a vertical moving plate in a porous medium. Uddin et.al.[18] investigated the influence of thermal radiation and heat generation//absorption on MHD heat transfer flow of a micropolar fluid past a wedge with hall and ion slip currents. A numerical investigation of MHD free convection flow of a non-Newtonian fluid past an impulsively started vertical plate in the presence of thermal diffusion and radiation absorption was conducted by Umamahesshwar [19]

In all these studies Soret/Dufour effects are assumed to be negligible. The Dufour effect is the energy flux due to a mass concentration gradient occurring as a coupled effect of irreversible processes. It is the reciprocal phenomenon to the Soret effect. Such effects are significant when density differences exist in the flow regime. For example, when species are introduced at a surface in a fluid domain, with density different (lower) than that of the surrounding fluid, both Soret and Dufour effects can be significant. Also, when heat and mass transfer occur simultaneously in a moving fluid, the relations between the fluxes and the driving potentials are of more intricate nature. It has been found that energy flux can be generated not only by temperature gradients but by composition gradients as well. When the composition of a mixture of molecules is non-uniform, the concentration gradient in any species of the mixture provides a driving potential for the diffusion of that species. The diffusing species flows from a region where it is highly concentrated to one where its concentration is low. An increase or decrease in the magnitude of concentration observed in passing from one point or moment to another is called composition gradient. The energy flux caused by a composition gradient is called the Dufour or diffusion-thermo effect. On the other hand, mass fluxes can also be created by temperature gradients and this is called the Soret or thermal-diffusion effect. Adrian [20] numerically investigated the heat mass transfer characteristics of natural convection about a vertical surface embedded in a saturated porous medium subjected to a magnetic field by taking into account the Dufour and Soret effects. Mahdy [21,22] studied MHD non-Darcian free convection from a vertical wavy surface embedded in a porous medium in the presence of the Soret and Dufour effect. Srinivasacharya and Reddy [23] investigated the Soret and Dufour effects on mixed convection heat and mass transfer in a micropolar fluid with heat and mass fluxes. Pal and Mondal $[24,25]$ examined the Soret and Dufour effect on MHD non-Darcy unsteady mixed convection heat and mass transfer over a stretching sheet. The study of diffusion the thermo effect on laminar mixed convection flow and heat transfer from a vertical sur- 
face with an induced magnetic field - was done by Hossain and Khatun [26]. Omowaye et al. [27] analyzed the Dufour and Soret effects on steady MHD convective flow of a fluid in a porous medium with temperature-dependent viscosity. Srinivasa Raju [28] examined the effects of Soret and Dufour on natural convective fluid flow past a vertical plate embedded in a porous medium in the presence of thermal radiation via FEM.

At a macroscopic level, it is well accepted that the boundary condition for a viscous fluid at a solid wall is one of no-slip condition i.e. the fluid velocity matches the velocity of the solid boundary. An assumption about no-slip condition is that it is not based on the physical principle but has been processed experimentally to be accurate for a number of macroscopic flows. In many applications, the particle adjacent to a solid surface no longer takes the velocity of the surface. The particle has a finite tangential velocity, it slips along the surface. The flow is called slip-flow. Sharma and Chaudhary [29] studied the effect of variable suction on transient free convective viscous incompressible flow past a vertical plate in a slip-flow regime. Khandelwal et al. [30] investigated the effects of couple stress on the flow through a porous medium with variable permeability in the slip-flow regime. Further, Sharma [31] studied the influence of periodic temperature and concentration on unsteady free convective viscous incompressible flow and heat transfer past a vertical plate in the slip-flow regime. The influence of magnetic field and wall slip conditions on the steady flow between a parallel flat wall and a long wavy wall with the Soret effect was observed by Muthuraj and Srinivas [32]. An analysis of steady flows in a viscous fluid with heat/mass transfer and the slip effect was done by Ellahi et al. [33]. Sreenivasulu et al. [34] investigated the thermal radiation effect on MHD boundary layer slip flow past a permeable exponential stretching sheet in the presence of joule heating and viscous dissipation. Velocity slip effects on heat and mass fluxes of MHD viscous-Ohmic dissipative flow over a stretching sheet with thermal radiation were studied by Kayalvizhi et al. [35].

Chemical reaction plays an important role in processes such as dying, distribution temperature and moisture over agricultural fields and groves of fruits, energy transfer in a wet cooling tower and flow in a desert cooler. The study of the chemical reaction is of great practical use in engineering and science. Chemical reaction, heat and mass transfer on MHD flow over a vertical stretching surface with heat source and thermal stratification effects are studied by Kandasamy et al. [36]. Chaudhary and Jha [37] investigated the effects of chemical reaction on MHD micropolar fluid flow past a vertical plate in the slip-flow regime. The effect of chemical reaction on free convective flow of a polar fluid through a porous medium in the presence of internal heat generation is discussed by Patil and Kulkarni [38]. Recently, Reddy et al. [39] studied the hall current and the Dufour effects on MHD flow of a micropolar fluid past a vertical plate in the presence of radiation absorption and chemical reaction. Pal et al. [40, 41] described the buoyancy and chemical reaction effects on MHD mixed convection heat and mass transfer in a porous medium with thermal radiation and joule heating.

Jonnadula Manjula, et al. [42] examined the influence of thermal radiation and chemical reaction on MHD flow, heat and mass transfer over a stretching surface. MHD flow, heat and mass transfer of a micropolar fluid over a nonlinear stretching sheet with variable micro inertia density, heat flux and chemical reaction in a non-Darcy porous medium were observed by Rawat et al. [43]. 
Joule heating, also known as Ohmic heating and resistive heating, is a process by which the passage of an electric current through a conductor releases heat. In all the above studies the permeability and porosity are usually taken as constant. Murthy and Feyen [44] studied the influence of variable permeability of the dispersion of a chemically reacting solute in a porous media. Hassanien et al. [45] analyzed the oscillatory hydro magnetic flow through a porous medium with variable permeability in the presence of free convection and mass transfer flow.

Thermal radiation is the emission of electromagnetic waves from all matter that has a temperature greater than absolute zero. It represents a conversion of thermal energy into electromagnetic energy. Recent developments in the area of nuclear power plants, gas turbines and the various propulsion devices for aircrafts, missile and space vehicles, hypersonic flights, gas cooled nuclear reactors and power plants for inter-planetary flights, have focused attention of researchers on thermal radiation as a mode of energy transfer, and emphasized the need for inclusion of radiative transfer in these process. Mahmoud [46] investigated the thermal radiation effects on an MHD flow micro polar fluid over a stretching surface with variable thermal conductivity. Ibrahim [47] studied the influence of viscous dissipation and radiation on the unsteady MHD mixed convection flow of a micropolar fluid. Free convection flow with thermal radiation and mass transfer past a moving vertical porous plate was investigated by Makinde [48]. Das [49] studied the slip effects on the hydro magnetic micro polar fluid flow and heat transfer over an inclined permeable plate with variable fluid properties and thermal radiation. Sreenivasulu et al.[50] studied the thermal radiation effects on the MHD boundary layer slip flow past a permeable exponential stretching sheet in the presence of joule heating and viscous dissipation.

\section{Definitions}

\section{MHD}

Magnetohydrodynamics is the study of the magnetic properties of electrically conducting fluid such as magneto-fluids; these include plasmas, liquid metals and electrolyte.

\section{POLAR FLUID}

A polar fluid is a fluid where the constituent molecules have a polarization. It could be a fluid of molecules that have a magnetic spin moment.

\section{OHMIC HEATING}

Joule heating, also known as Ohmic heating and resistive heating, is the process by which the passage of an electric current through a conductor releases heat.

\section{DUFOUR EFFECT}

The Dufour effect is the energy flux due to a mass concentration gradient occurring as a coupled effect of irreversible processes.

\section{BUOYANCY}

Buoyancy is an upward force exerted by a fluid that opposes the weight of an immersed object.

In the present analysis, we proposed a study of the effect of first order chemical reaction, the Dufour effect and thermal radiation on an unsteady MHD Newtonian and polar fluid flow past an infinite vertical porous plate in slip flow regime. We also consider the presence of heat source, Ohmic heating and viscous dissipation. 


\section{Formulation of the problem for a Newtonian Fluid}

We consider an unsteady two-dimensional hydro magnetic mixed convective boundary layer flow of a viscous, incompressible, two dimensional slip flow in an optically thin environment, past a semi-infinite vertical moving plate embedded in a porous medium of variable permeability with variable mass diffusion, in the presence of thermal radiation. We assume that no electric field is present and induced magnetic field is negligible. The $X$-axis is taken along the plate in a vertically upward direction and the $Y$-axis is normal to it. Due to the semi-infinite plane surface assumption, the flow variables are functions of the normal distance $Y$ and $t$ only, the time-dependent suction velocity is assumed normal to the plate. The plate is having a variable temperature and concentration which varies with time. Now under the usual Boussinesq's approximation, the governing boundary layer equations in the non-dimensional form are:

$$
\begin{gathered}
\frac{\partial u}{\partial t}-\left(1+\varepsilon A e^{n t}\right) \frac{\partial u}{\partial y}=\frac{d U_{\infty}}{d t}+\frac{\partial^{2} u}{\partial y^{2}}+G_{r} \theta+G_{m} C+N\left(U_{\infty}-u\right) \\
\frac{\partial \theta}{\partial t}-\left(1+\varepsilon A e^{n t}\right) \frac{\partial \theta}{\partial y}=\frac{1}{P_{r}} \frac{\partial^{2} \theta}{\partial y^{2}}+E_{c}\left(\frac{\partial u}{\partial y}\right)^{2}-S \theta+N E_{c} u^{2}+D_{u} \frac{\partial^{2} c}{\partial y^{2}}-F \theta \\
\frac{\partial C}{\partial t}-\left(1+\varepsilon A e^{n t}\right) \frac{\partial C}{\partial y}=\frac{1}{S_{c}} \frac{\partial^{2} C}{\partial y^{2}}-\gamma C
\end{gathered}
$$

where $u, v$ are the velocity components in $X, Y$ directions, respectively, $t$-the time parameter $\varepsilon$ and $\mathrm{n}$ is the scalar constant. The third and fourth terms on the right hand side of the momentum Eq. (2.1) denote the thermal and concentration buoyancy effects, respectively. Also, the second and fourth terms on right hand side of the energy Eq. (2.2) represent viscous dissipation and the Joule heating effects, respectively. In addition, the third and fifth terms denote heat absorption and the Dufour effect, respectively. $\mathrm{G}_{r}, \mathrm{G}_{m}, \mathrm{P}_{r}, \mathrm{~F}, \mathrm{E}_{c}$ , $\mathrm{S}_{c}, \mathrm{~S}$ and $\mathrm{D}_{u}$ are the thermal Grashof number, the Solutal Grashof Number, the Prandtl Number, the radiation parameter, the Eckert number, the Schmidt number, the chemical reaction rate of the heat source parameter and the Dufour effect parameter, respectively.

$\mathrm{N}=\mathrm{M}+1 / \mathrm{K}_{0}$ where $\mathrm{M}$ is the magnetic field parameter, and $\mathrm{K}_{0}$ is the permeability parameter, respectively.

The suction velocity to the plate is assumed in the form

$$
v^{\prime}=-V_{0}\left(1+\varepsilon A e^{n^{\prime} t^{\prime}}\right)
$$

where $\mathrm{A}$ is a real positive constant and $\varepsilon$ is small such that $\varepsilon<<1, \mathrm{~A}<<1$, and $\mathrm{V}_{0}$ is a non-zero positive constant. The negative sign indicates that the suction is towards the plate.

The corresponding dimensionless boundary conditions are:

$$
u=1+h \frac{\partial u}{\partial y}, \theta=1+\varepsilon e^{n t}, C=1+\varepsilon e^{n t} a t y=0
$$




$$
U \rightarrow U_{\infty}, \theta \rightarrow 0, C \rightarrow 0 \text { as } y \rightarrow \infty
$$

The above systems are coupled and linear partial differential equations and these can be reduced to a set of ordinary differential equations with the help of the perturbation technique, which can be solved analytically. Perturbation theory is applicable if the problem at hand cannot be solved exactly, but can be formulated by adding a "small" term to the mathematical description of the exactly solvable problem. Perturbation theory leads to an expression for the desired solution in terms of a formal power series in a "small" parameter - known as a perturbation series - that quantifies the deviation from the exactly solvable problem ( Dyke V.M.[51]). The equations are solved manually. All the coefficients are calculated numerically and the graphs are plotted using MS Excel and C language.

This can be done by representing the velocity, temperature and concentration of the fluid in the neighborhood of the plate as:

$$
\begin{gathered}
u(y, t)=u_{0}(y)+\varepsilon e^{n t} u_{1}(y)+o\left(\varepsilon^{2}\right)+\cdots \\
\theta(y, t)=\theta_{0}(y)+\varepsilon e^{n t} \theta_{1}(y)+o\left(\varepsilon^{2}\right)+\cdots \\
C(y, t)=C_{0}(y)+\varepsilon e^{n t} C_{1}(y)+o\left(\varepsilon^{2}\right)+\cdots
\end{gathered}
$$

We assume that permeability of the porous medium is variable so $K=K_{0}\left(1+\varepsilon e^{n t}\right)$, where $K_{0}>0$ corresponds to the permeability of the porous medium. By substituting Eq. (2.4) in Eq. 2.1-2.3, equating the harmonic and non-harmonic terms, and neglecting the higher order terms of $o\left(\varepsilon^{2}\right)$, we obtain:

$$
\begin{gathered}
u_{0}^{\prime \prime}+u_{0}^{\prime}-N u_{0}=-N-G_{r} \theta_{0}-G_{m} C_{0} \\
u_{1}^{\prime \prime}+u_{1}^{\prime}-(n+N) u_{1}=-(N+n)-G_{r} \theta_{1}-G_{m} C_{1}-A u_{0}^{\prime} \\
\theta_{0}^{\prime \prime}+P_{r} \theta_{0}^{\prime}-P_{r}(S+F) \theta_{0}=-P_{r} E c\left(u_{0}^{\prime}\right)^{2}-P_{r} N E_{C} u_{0}^{2}-P_{r} D_{u} C_{0}^{\prime \prime} \\
\theta_{1}^{\prime \prime}+P_{r} \theta_{1}^{\prime}-(F+n+S) P_{r} \theta_{1}=-P_{r} A \theta_{0}^{\prime}-2 P_{r} E_{c} u_{0}^{\prime} u_{1}^{\prime}-2 P_{r} N E_{c} u_{0} u_{1}-P_{r} D_{u} C_{1}^{\prime \prime} \\
C_{0}^{\prime \prime}+S_{c} C_{0}^{\prime}=S_{c} \cdot \gamma \cdot C_{0} \\
C_{1}^{\prime \prime}+S_{c} C_{1}^{\prime}-S_{c} \cdot(\gamma+n) \cdot C_{1}=-A S_{c} C_{0}^{\prime}
\end{gathered}
$$

where prime denotes ordinary differentiation with respect to $y$.

The corresponding boundary conditions ( Chaudhary and Jha [37] )can be written as:

$$
u_{0}=1+h \frac{\partial u_{0}}{\partial y}, u_{1}=h \frac{\partial u_{0}}{\partial y} \theta_{0}=1, \theta_{1}=0, C_{0}=1, C_{1}=0 \text { at } y=0
$$




$$
u_{0}=1, u_{1}=1, \theta_{0}=0, \theta_{1}=0, C_{0}=0, C_{1}=0 \text { at } y \rightarrow \infty
$$

Equations 2.5-2.10 are still coupled and non-linear, whose exact solution has not been found yet. So we expand $u_{0}, u_{1}, \theta_{0}, \theta_{1}, C_{0}, C_{1}$ in terms of $E_{c}$ in the following form, as the Eckert number is very small for incompressible flows. We use

$$
F(y)=F_{0}(y)+E_{c} F_{1}(y)+o\left(E_{c}\right)^{2}+\ldots .
$$

where $\mathrm{F}$ stands for any $u_{0}, u_{1}, \theta_{0}, \theta_{1}, C_{0}, C_{1}$ Substituting Eq. (2.12) in Eq. 2.5-2.10, equating the coefficients of $E_{c}$ to zero, neglecting the terms in $E_{c}^{2}$ and higher order, and solving the equations under the corresponding boundary conditions, we obtain the velocity, temperature and concentration distributions for the Newtonian fluid in the boundary layer as:

$$
\begin{aligned}
& u=A_{5} e^{-m_{3} y}+A_{4} e^{-m_{2} y}+A_{3} e^{-m_{1} y}+1+E_{c}\left(A_{19} e^{-m_{3} y}+A_{6} e^{-m_{2} y}+A_{7} e^{-2 m_{3} y}+A_{8}+A_{9} e^{-2 m_{2} y}\right. \\
& +A_{10} e^{-2 m_{1} y}+A_{11} e^{-\left(m_{2}+m_{3}\right) y}+A_{12} e^{-\left(m_{1}+m_{3}\right) y}+A_{13 a} e^{-m_{2} y}+y A_{13 b} e^{-m_{2} y}+A_{14 a} e^{-m_{3} y}+y A_{14 b} e^{-m_{3} y} \\
& \left.+A_{15} e^{-m_{1} y}+A_{16} e^{-\left(m_{1}+m_{2}\right) y}\right)+\varepsilon e^{n t}\left(A_{27} e^{-m_{8} y}+A_{20} e^{-m_{6} y}+A_{21} e^{-m_{2} y} A_{22} e^{-m_{7} y}\right. \\
& +A_{23} e^{-m_{1} y}+A_{24} e^{-m_{3} y}+1+E c\left(A_{60} e^{-m_{8} y}+A_{34} e^{-m_{3} y}+A_{35} e^{-m_{2} y}+A_{36} e^{-2 m_{3} y}\right. \\
& +A_{37}+A_{38} e^{-2 m_{2} y}+A_{39} e^{-2 m_{1} y} A_{40} e^{-\left(m_{2}+m_{3}\right) y}+A_{41} e^{-\left(m_{1}+m_{3}\right) y}+y A_{42} e^{-m_{2} y}+y A_{43} e^{-m_{3} y} \\
& +A_{44} e^{-m_{1} y}+A_{45} e^{-\left(m_{2}+m_{1}\right) y}+A_{46} e^{-m_{6} y}+A_{47} e^{-\left(m_{8}+m_{3}\right) y}+A_{48} e^{-\left(m_{6}+m_{3}\right) y} A_{49} e^{-\left(m_{7}+m_{3}\right) y} \\
& +A_{50} e^{-\left(m_{2}+m_{8}\right) y}+A_{51} e^{-\left(m_{2}+m_{6}\right) y}+A_{52} e^{-\left(m_{1}+m_{6}\right) y}+A_{53} e^{-\left(m_{1}+m_{8}\right) y}+A_{54} e^{-\left(m_{1}+m_{7}\right) y} \\
& \left.\left.+A_{55} e^{-\left(m_{2}+m_{7}\right) y} A_{56}+y A_{57} e^{-m_{2} y}\right)\right) \\
& C=e^{-m_{1} y}+\varepsilon e^{n t}\left(A_{33} e^{-m_{7} y}+A_{32} e^{-m_{1} y}\right)
\end{aligned}
$$

$$
\begin{aligned}
& \theta=A_{2} e^{-m_{2} y}+A_{1} e^{-m_{1} y}+E c\left(f_{11} e^{-m_{2} y}+f_{1} e^{-2 m_{3} y}+f_{2}+f_{3} e^{-2 m_{2} y}+f_{4} e^{-2 m_{1} y}\right. \\
& +f_{5} e^{-\left(m_{2}+m_{3}\right) y}+f_{6} e^{-\left(m_{1}+m_{3}\right) y}+f_{7} e^{-m_{2} y}+y f_{7 b} e^{-m_{2} y}+f_{8} e^{-m_{3} y}+f_{9} e^{-m_{1} y} \\
& \left.+f_{10} e^{-\left(m_{1}+m_{2}\right) y}\right)+\varepsilon e^{n t}\left(f_{16} e^{-m_{6} y}+f_{13} e^{-m_{2} y}+f_{14} e^{-m_{1} y}+f_{15} e^{-m_{7} y}+E c\left(f_{44} e^{-m_{6} y}\right.\right. \\
& +f_{17} e^{-\left(m_{3}+m_{8}\right) y}+f_{18} e^{-\left(m_{3}+m_{6}\right) y}+f_{19} e^{-\left(m_{3}+m_{1}\right) y}+f_{20} e^{-\left(m_{3}+m_{7}\right) y}+f_{21} e^{-\left(m_{3}+m_{2}\right) y} \\
& +f_{22} e^{-m_{3} y}+f_{23} e^{-\left(m_{2}+m_{8}\right) y}+f_{24} e^{-\left(m_{2}+m_{6}\right) y}+f_{25} e^{-m_{2} y} \\
& +f_{26} e^{-\left(m_{1}+m_{6}\right) y}+f_{27} e^{-\left(m_{1}+m_{8}\right) y}+f_{28} e^{-\left(m_{2}+m_{1}\right) y}+f_{29} e^{-\left(m_{1}+m_{7}\right) y} \\
& +f_{30} e^{-m_{1} y}+f_{31} e^{-\left(m_{7}+m_{2}\right) y}+f_{32} e^{-m_{2} y}+f_{33} e^{-2 m_{3} y}+f_{34} e^{-2 m_{2} y}+f_{35} \\
& +f_{36} e^{-2 m_{1} y}+f_{37} e^{-\left(m_{2}+m_{3}\right) y}+f_{38} e^{-\left(m_{3}+m_{1}\right) y}+f_{39} e^{-m_{2} y}+f_{40} e^{-m_{2} y} y+f_{41} e^{-m_{3} y} \\
& \left.\left.+f_{42} e^{-m_{1} y}+f_{43} e^{-\left(m_{1}+m_{2}\right) y}\right)\right)
\end{aligned}
$$

where

$$
m_{1}=\frac{S c+\sqrt{S c^{2}+4 \gamma S c}}{2}, m_{2}=\frac{\operatorname{Pr}+\sqrt{\operatorname{Pr}^{2}+4 \operatorname{Pr}(F+S)}}{2}, m_{4}=\frac{\operatorname{Pr}-\sqrt{\operatorname{Pr}^{2}+4 \operatorname{Pr}(F+S)}}{2},
$$




$$
\begin{gathered}
m_{6}=\frac{\operatorname{Pr}+\sqrt{\operatorname{Pr}^{2}+4 \operatorname{Pr}(F+S+n)}}{2}, m_{7}=\frac{S c+\sqrt{S c^{2}+4 S c(\gamma+n)}}{2}, m_{8}=\frac{1+\sqrt{1+4(N+n)}}{2}, \\
A_{1}=\frac{-\operatorname{Pr} D u m_{1}^{2}}{m_{1}^{2}-m_{1} \operatorname{Pr}-\operatorname{Pr}(F+S)}, A_{2}=1-A_{1}, m_{3}=\frac{1+\sqrt{1+4 N}}{2}, m_{5}=\frac{1-\sqrt{1+4 N}}{2}, \\
A_{4}=\frac{-G r A_{2}}{m_{2}^{2}-m_{2}-N}, A_{3}=\frac{-G r A_{1}-G c}{m_{1}^{2}-m_{1}-N}, A_{5}=\frac{-1}{\left(h m_{3}+1\right)}\left[A_{4}\left(h m_{2}+1\right)+A_{3}\left(h m_{1}+1\right)\right]
\end{gathered}
$$

\section{Skin-friction}

The boundary layer produces a drag on the plate due to the viscous stresses, which are developed at the wall. The viscous stress at the surface of the plate is given by

$$
\tau_{x}=\left(\frac{\partial u_{0}}{\partial y}\right)_{y=0}+E_{c}\left(\frac{\partial u_{1}}{\partial y}\right)_{y=0}
$$

\section{Heat transfer rate}

The Nusselt number $\mathrm{N}_{u}$ is often used to determine heat transfer. The non-dimensional heat flux at the plate $\mathrm{y}=0$ in terms of $\mathrm{N}_{u}$ is given by

$$
N u=\left(\frac{\partial \theta_{0}}{\partial y}\right)_{y=0}+E_{c}\left(\frac{\partial \theta_{1}}{\partial y}\right)_{y=0}
$$

\section{Mass flux}

The Sharewood number $S_{h}$ is denoted mass transfer. The non-dimensional mass flux at the plate $\mathrm{y}=0$ in terms of $\mathrm{S}_{h}$ is given by

$$
S_{h}=\left(\frac{\partial C_{0}}{\partial y}\right)_{y=0}+E_{c}\left(\frac{\partial c_{1}}{\partial y}\right)_{y=0}
$$

\section{Formulation of the problem for polar fluid}

We consider an unsteady two-dimensional hydro magnetic mixed convective boundary layer flow of a polar electrically conducting, incompressible, viscous two-dimensional slip flow in an optically thin environment, past a semi-infinite vertical moving plate embedded in a porous medium of variable permeability with mass diffusion, in the presence of thermal radiation. We assume that no electric field is present and that the induced magnetic field is negligible. The $x^{\prime}$-axis is taken along the plate in the vertically upward direction and the y'-axis is normal to it. Due to the semi-infinite plane surface assumption, the flow variables are functions of the normal distance y' and t' only. A time depend suction velocity is assumed normal to the plate. The plate has a variable temperature and concentration which varies with time. Now under the usual Boussinesq's approximation, the governing boundary layer equations in the non-dimensional form are:

(3.1) $\frac{\partial u}{\partial t}-\left(1+\varepsilon A e^{n t}\right) \frac{\partial u}{\partial y}=\frac{d U_{\infty}}{d t}+(1+\alpha) \frac{\partial^{2} u}{\partial y^{2}}+G_{r} \theta+G_{m} C+N\left(U_{\infty}-u\right)+2 \alpha \frac{\partial \Omega}{\partial y}$ 


$$
\begin{gathered}
\frac{\partial \Omega}{\partial t}-\left(1+A \varepsilon e^{n t}\right) \frac{\partial \Omega}{\partial y}=\frac{1}{\beta} \frac{\partial^{2} \Omega}{\partial y^{2}} \\
\frac{\partial \theta}{\partial t}-\left(1+\varepsilon A e^{n t}\right) \frac{\partial \theta}{\partial y}=\frac{1}{P_{r}} \frac{\partial^{2} \theta}{\partial y^{2}}+E_{c}\left(\frac{\partial u}{\partial y}\right)^{2}-S \theta-F \theta+N E_{C} u^{2}+D_{u} \frac{\partial^{2} c}{\partial y^{2}} \\
\frac{\partial C}{\partial t}-\left(1+\varepsilon A e^{n t}\right) \frac{\partial C}{\partial y}=\frac{1}{S_{c}} \frac{\partial^{2} C}{\partial y^{2}}-\gamma C
\end{gathered}
$$

The corresponding dimensionless boundary conditions are:

$$
u=1+h \frac{\partial u}{\partial y}, \theta=1+\varepsilon e^{n t}, \omega=-n \frac{\partial u}{\partial y}, C=1+\varepsilon e^{n t} \text { at } y=0
$$

$$
U \rightarrow U_{\infty}, \theta \rightarrow 0, \omega \rightarrow 0, C \rightarrow 0 \text { as } y \rightarrow \infty
$$

here $\mathrm{u}, \mathrm{v}$ are velocity components, $\Omega$ is the angular velocity component, $\rho$ is density, $v=$ $\frac{\mu}{\rho}$ is kinematic viscosity, $v_{r}$ is rotational kinematic viscosity, $\gamma=\frac{\left(c_{a}+c_{d}\right)}{I}\left(\mathrm{c}_{a}, \mathrm{c}_{d}\right.$ are coupled stress viscosities, I is a scalar constant equal to that of the moment of inertia unit mass). $\Omega=\frac{\Omega^{\prime} v}{V_{0}^{2}} \alpha=\frac{v_{r}}{v} \beta=I \frac{v}{\gamma}$

The value $n=0$ corresponds to the case where the particle density is sufficiently large so that microelements close to the wall are unable to rotate. The value $n=0.5$ is indicative of the weak concentration, and when $n=1$ the flows are believed to represent turbulent boundary layers (Rees and Bossom [52]).

\section{Solution of the problem}

The above systems are coupled and linear partial differential equations and these can be reduced to a set of ordinary differential equations, which can be solved analytically. This can be done by representing the velocity, temperature and concentration of the fluid in the neighborhood of the plate as:

$$
\begin{gathered}
u(y, t)=u_{0}(y)+\varepsilon e^{n t} u_{1}(y)+o\left(\varepsilon^{2}\right)+\cdots \\
\theta(y, t)=\theta_{0}(y)+\varepsilon e^{n t} \theta_{1}(y)+o\left(\varepsilon^{2}\right)+\cdots \\
C(y, t)=C_{0}(y)+\varepsilon e^{n t} C_{1}(y)+o\left(\varepsilon^{2}\right)+\cdots
\end{gathered}
$$

Substituting Eq. (3.6) in Eq. 3.1-3.4 and equating harmonic and non-harmonic terms and neglecting the higher order terms of $o\left(\varepsilon^{2}\right)$, we obtain:

$$
(1+\alpha) u_{0}^{\prime \prime}+u_{0}^{\prime}-N u_{0}=-N-G_{r} \theta_{0}-G_{m} C_{0}-2 \alpha \Omega_{0}^{\prime}
$$




$$
\begin{gathered}
\Omega_{0}^{\prime \prime}+\beta \Omega_{0}^{\prime}=0 \\
(1+\alpha) u_{1}^{\prime \prime}+u_{1}^{\prime}-(n+N) u_{1}=-(N+n)-G_{r} \theta_{1}-G_{m} C_{1}-A u_{0}^{\prime}-2 \alpha \Omega_{1}^{\prime} \\
\Omega_{1}^{\prime \prime}+\beta \Omega_{1}^{\prime}-n \beta \Omega_{1}=-A \beta \Omega_{0}^{\prime} \\
\theta_{0}^{\prime \prime}+P_{r} \theta_{0}^{\prime}-P_{r}(S+F) \theta_{0}=-P_{r} E_{c}\left(u_{0}^{\prime}\right)^{2}-P_{r} N E_{C} u_{0}^{2}-P_{r} D_{u} C_{0}^{\prime \prime} \\
\theta_{1}^{\prime \prime}+P_{r} \theta_{1}^{\prime}-(F+n+S) P_{r} \theta_{1}=-P_{r} A \theta_{0}^{\prime}-2 P_{r} E_{c} u_{0}^{\prime} u_{1}^{\prime}-2 P_{r} N E_{c} u_{0} u_{1}-P_{r} D_{u} C_{1}^{\prime \prime} \\
C_{0}^{\prime \prime}+S_{c} C_{0}^{\prime}=S_{c} \cdot \gamma \cdot C_{0} \\
C_{1}^{\prime \prime}+S_{c} C_{1}^{\prime}-S_{c} \cdot(\gamma+n) \cdot C_{1}=-A S_{c} C_{0}^{\prime}
\end{gathered}
$$

The corresponding boundary conditions can be written as:

$$
\begin{aligned}
& u_{0}=1+h \frac{\partial u_{0}}{\partial y}, u_{1}=h \frac{\partial u_{0}}{\partial y} \theta_{0}=1, \theta_{1}=0, C_{0}=1, C_{1}=0, \\
& \Omega_{0}=-n \frac{\partial u_{0}}{\partial y}, \Omega_{1}=-n \frac{\partial u_{1}}{\partial y} a t, \\
& y=0 u_{0}=1, u_{1}=1, \theta_{0}=0, \theta_{1}=0, C_{0}=0, C_{1}=0, \Omega_{0} \rightarrow 0, \Omega_{1} \rightarrow \text { at } y \rightarrow \infty
\end{aligned}
$$

The Eq. (5.2-5.9) are still coupled and non-linear, whose exact solution has not yet been found. So we expand $u_{0}, u_{1}, \theta_{0}, \theta_{1}, C_{0}, C_{1}$ in terms of $E_{c}$ in the following form, as the Eckert number is very small for incompressible flows.

$$
F(y)=F_{0}(y)+E_{c} F_{1}(y)+o\left(E_{c}\right)^{2}+\cdots
$$

where $F$ stands for any $u_{0}, u_{1}, \theta_{0}, \theta_{1}, C_{0}, C_{1}$ Substituting Eq. (3.16) in Eq. (3.7)-(3.14), equating the coefficients of $E_{c}$ to zero and neglecting the terms in $E_{c}^{2}$ and higher order, after solving these equations, we obtain the velocity, temperature, angular momentum and concentration distributions in the boundary layer as:

$$
\text { (3.17) }
$$

$$
\begin{aligned}
& u=T_{5} e^{-B_{1} y}+T_{1} e^{-m_{2} y}+T_{2} e^{-m_{1} y}+1+c_{2} T_{3} e^{-\beta y}+E_{c}\left(T_{24} e^{-B_{1} y}+T_{6} e^{-m_{2} y}+T_{7} e^{-2 B_{1} y}+T_{8} e^{-2 m_{2} y}\right. \\
& +T_{9} e^{-2 m_{1} y}+T_{10} e^{-2 \beta y}+T_{11} e^{-\left(m_{2}+B_{1}\right) y}+T_{12} e^{-\left(m_{1}+B_{1}\right) y}+T_{13} e^{-\left(B_{1}+\beta\right) y}+T_{14} e^{-\left(m_{2}+m_{1}\right) y}+T_{15} e^{-\left(m_{2}+\beta\right) y} \\
& \left.+T_{16} e^{-\left(m_{1}+\beta\right) y}+T_{17} e^{-B_{1} y}+T_{18} e^{-m_{2} y}+T_{19} e^{-m_{1} y}+T_{20} e^{-\beta y}+T_{21}+y T_{22} e^{-m_{2} y}+T_{23} c_{2} e^{-\beta y}\right)+\varepsilon e^{n t}\left(T_{42} e^{-B_{2} y}\right. \\
& +T_{29} e^{-B_{1} y}+T_{30} e^{-m_{2} y}+T_{32} e^{-\beta y}+T_{33} e^{-m_{6} y}+T_{35} e^{-m_{7} y}+T_{36} e^{-m_{1} y} \\
& +1+T_{40} T_{39} e^{-\beta_{1} y}+E c\left(T_{80} e^{-B_{2} y}+T_{46} e^{-B_{1} y}+T_{47} e^{-m_{2} y}+T_{48} e^{-2 B_{1} y}+T_{49} e^{-2 m_{2} y}+T_{50} e^{-2 m_{1} y}+T_{51} e^{-2 \beta y}\right. \\
& +T_{52} e^{-\left(m_{1}+B_{1}\right) y}+T_{53} e^{-\left(B_{1}+\beta\right) y}+T_{54} e^{-\left(m_{2}+m_{1}\right) y}+T_{55} e^{-\left(m_{2}+\beta\right) y} \\
& +T_{56} e^{-\left(\beta+m_{1}\right) y}+T_{57} e^{-m_{1} y}+T_{58} e^{-\beta y}+y T_{59} e^{-m_{2} y}+T_{60} e^{-m_{6} y}+T_{61} \\
& +T_{62} e^{-\left(m_{2}+B_{1}\right) y}+T_{63} e^{-\left(B_{2}+B_{1}\right) y}+T_{64} e^{-\left(m_{6}+B_{1}\right) y}+T_{65} e^{-\left(m_{7}+B_{1}\right) y}+T_{66} e^{-\left(2 \beta_{1}\right) y} T_{67} e^{-\left(m_{2}+B_{2}\right) y} \\
& +T_{68} e^{-\left(m_{2}+m_{6}\right) y}+T_{69} e^{-\left(m_{2}+m_{7}\right) y}+T_{70} e^{-\left(m_{2}+\beta_{1}\right) y}+T_{71} e^{-\left(B_{2}+m_{1}\right) y} \\
& +T_{72} e^{-\left(m_{1}+m_{7}\right) y}+T_{73} e^{-\left(m_{1}+\beta_{1}\right) y}+T_{74} e^{-\left(B_{2}+\beta\right) y}+T_{75} e^{-\left(\beta+m_{6}\right) y}+T_{76} e^{-\left(\beta+m_{7}\right) y} \\
& \left.\left.+T_{77} e^{-\left(\beta+\beta_{1}\right) y}+T_{78} e^{-m_{7} y}+T_{79} e^{-\left(m_{1}+m_{6}\right) y}+w_{1} c_{4} e^{-\beta_{1} y}+w_{4} e^{-\beta y}\right)\right)
\end{aligned}
$$




$$
\begin{gathered}
\Omega=c_{2} e^{-\beta y}+E_{c}\left(c_{3} e^{-\beta y}\right)+\varepsilon e^{n t}\left(T_{39} e^{-\beta_{1} y}+T_{38} e^{-\beta y}+E_{c}\left(w_{1} e^{-\beta_{1} y}+w_{2} e^{-\beta y}\right)\right) \\
C=e^{-m_{1} y}+\varepsilon e^{n t}\left(A_{33} e^{-m_{7} y}+A_{32} e^{-m_{1} y}\right)
\end{gathered}
$$

(3.18)

$$
\begin{aligned}
& \theta=A_{2} e^{-m_{2} y}+A_{1} e^{-m_{1} y}+E c\left(f_{20} e^{-m_{2} y}+f_{5} e^{-2 B_{1} y}+f_{6} e^{-2 m_{2} y}+f_{7} e^{-2 m_{1} y}+f_{8} e^{-2 \beta y}\right. \\
& +f_{9} e^{-\left(m_{2}+B_{1}\right) y}+f_{10} e^{-\left(m_{1}+B_{1}\right) y}+f_{11} e^{-\left(\beta+B_{1}\right) y}+f_{12} e^{-\left(m_{1}+m_{2}\right) y} \\
& \left.+f_{13} e^{-\left(m_{2}+\beta\right) y}+f_{14} e^{-\left(m_{1}+\beta\right) y}+f_{15} e^{-B_{1} y}+y f_{16 b} e^{-m_{2} y}+f_{16 a} e^{-m_{2} y}+f_{17} e^{-m_{1} y}+f_{18} e^{-\beta y}+f_{19}\right)+\varepsilon e^{n t}\left(f_{16} e^{-m_{6} y}\right. \\
& +f_{13} e^{-m_{2} y}+f_{14} e^{-m_{1} y}+f_{15} e^{-m_{7} y}+E c\left(f_{52} e^{-m_{6} y}+f_{17} e^{-\left(B_{1}+B_{2}\right) y}+f_{18} e^{-2 B_{1} y}+f_{19} e^{-\left(m_{2}+B_{1}\right) y}+f_{20} e^{-\left(B_{1}+\beta\right) y}\right. \\
& +f_{21} e^{-\left(B_{1}+m_{6}\right) y}+f_{22} e^{-\left(B_{1}+m_{7}\right) y}+f_{23} e^{-\left(m_{1}+B_{1}\right) y}+f_{24} e^{-\left(B_{1}\right) y}+f_{25} e^{-2 \beta_{1} y}+f_{26} e^{-\left(m_{2}+B_{2}\right) y} \\
& +f_{27} e^{-2 m_{2} y}+f_{28} e^{-\left(m_{2}+\beta\right) y}+f_{29} e^{-\left(m_{2}+m_{6}\right) y}+f_{30} e^{-\left(m_{7}+m_{2}\right) y}+f_{31} e^{-\left(m_{1}+m_{2}\right) y}+f_{32} e^{-m_{2} y} \\
& +f_{33} e^{-\left(\beta_{1}+m_{2}\right) y}+f_{34} e^{-\left(m_{1}+B_{2}\right) y}+f_{35} e^{-\left(m_{7}+m_{1}\right) y}+f_{36} e^{-2 m_{1} y}+f_{37} e^{-m_{1} y}+f_{38} e^{-\left(m_{1}+\beta_{1}\right) y} \\
& +f_{39} e^{-\left(B_{2}+\beta\right) y}+f_{40} e^{-\beta y}+f_{41} e^{-\left(m_{1}+\beta\right) y}+f_{42} e^{-\left(\beta+m_{6}\right) y}+f_{43} e^{-\left(\beta+m_{7}\right) y} \\
& +f_{44} e^{-\left(\beta+\beta_{1}\right) y}+f_{45} e^{-B_{2} y}+f_{46} e^{-2 \beta y}+f_{47} e^{-m_{6} y} \\
& \left.\left.+f_{48} e^{-m_{7} y}+y f_{49} e^{-m_{2} y}+f_{50}+f_{51} e^{-\left(m_{1}+m_{6}\right) y}\right)\right)
\end{aligned}
$$

Where

$$
\begin{aligned}
& m_{1}=\frac{S c+\sqrt{S c^{2}+4 \gamma S c}}{2}, m_{2}=\frac{\operatorname{Pr}+\sqrt{\operatorname{Pr}^{2}+4 \operatorname{Pr}(F+S)}}{2}, m_{4}=\frac{\operatorname{Pr}-\sqrt{\operatorname{Pr}^{2}+4 \operatorname{Pr}(F+S)}}{2}, \\
& m_{6}=\frac{\operatorname{Pr}+\sqrt{\operatorname{Pr}^{2}+4 \operatorname{Pr}(F+S+n)}}{2}, m_{7}=\frac{S c+\sqrt{S c^{2}+4 S c(\gamma+n)}}{2}, \\
& B_{2}=\frac{1+\sqrt{1+4(N+n)}(1+\alpha)}{2(1+\alpha)}, A_{1}=\frac{-\operatorname{Pr} D_{u m}^{2}}{m_{1}^{2}-m_{1} \operatorname{Pr}-\operatorname{Pr}(F+S)}, A_{2}=1-A_{1} \text {, } \\
& \beta_{1}=\frac{\beta+\sqrt{\beta^{2}+4 \beta n}}{2}, B_{1}=\frac{1+\sqrt{1+4 N(1+\alpha)}}{2(1+\alpha)}, m_{5}=\frac{1-\sqrt{1+4 N}}{2}, \\
& T_{1}=\frac{-G r A_{2}}{(1+\alpha) m_{2}^{2}-m_{2}-N}, T_{2}=\frac{-G r A_{1}-G c}{(1+\alpha) m_{1}^{2}-m_{1}-N}, T_{3}=\frac{2 \alpha \beta}{(1+\alpha) \beta^{2}-\beta-N} \\
& T_{5}=\frac{-1}{\left(h B_{1}+1\right)}\left[1+T_{1}\left(h m_{2}+1\right)+T_{2}\left(h m_{1}+1\right)\right]-\frac{1}{\left(h B_{1}+1\right)}\left[T_{3} c_{2}(h \beta+1)\right] \\
& c_{2}=\frac{n\left[T_{4} B_{1}+T_{1} m_{2}+T_{2} m_{1}\right]}{\left[1+\frac{(1+h \beta) n T_{3} B_{1}}{1+h B_{1}}-n T_{3} \beta\right]}, T_{4}=\frac{-1}{\left(h B_{1}+1\right)}\left[1+T_{1}\left(h m_{2}+1\right)+T_{2}\left(h m_{1}+1\right)\right]
\end{aligned}
$$

Given the velocity field in the boundary layer, we can now calculate the skin friction coefficient $\mathrm{C}_{f}$ at the wall which is given by

$$
\tau_{\omega}=\left.(\mu+\Lambda) \frac{\partial u^{*}}{\partial y^{*}}\right|_{y^{*}=0}+\left.\Lambda \Omega^{*}\right|_{y^{*}=0}
$$




$$
C_{f}=\frac{2 T_{W}^{*}}{\rho U_{0} V_{0}}=2\left[1+(1-n) \alpha_{v}\right] u^{\prime}(0)
$$

The couple stress coefficient $\mathrm{C}_{m}$ at the plate is written as

$$
\begin{gathered}
C_{\Omega}=\left.\gamma \frac{\partial \Omega^{*}}{\partial y^{*}}\right|_{y^{*}=0} \\
C_{m}=\frac{M_{W}}{\mu J U_{0}}=\left(1+\frac{\alpha_{v}}{2}\right) .
\end{gathered}
$$

The rate of heat transfer i.e. heat flux at the wall in terms of Nusselt number $\left(N_{u}\right)$ is given in the non-dimensional form by

$$
N_{u}=-\left[\left(\frac{\partial T_{0}}{\partial y}\right)_{y=0}+\varepsilon\left(\frac{\partial T_{1}}{\partial y}\right)_{y=0}\right] .
$$

\section{RESULT AND DISCUSSION}

A numerical evaluation for the analytical solution to the problem is performed and the results are illustrated graphically. To show interesting features of the significant physical parameter on the velocity, angular velocity, temperature, concentration, skin friction, the Nusselt number and the Sharewood number on the distribution of the Newtonian and polar fluid along an infinite porous plate, throughout the computations we employ the thermal Grashof number $\left(G_{r}\right)=5$, the Solutal Grashof number $\left(G_{c}\right)=5$, variable permeability $\left(K_{0}\right)=$ .5 , the chemical reaction parameter $\gamma=.5$, the heat source parameter $(\mathrm{S})=1$, the Dufour effect parameter $\left(D_{u}\right)=.5$, the radiation parameter $(\mathrm{F})=1$, the Hartmann number $(\mathrm{M})=5$, the Schmidt number $\left(\mathrm{S}_{c}\right)=.33, \mathrm{n}=1$, the Prandtl number $\left(\mathrm{P}_{r}\right)=.71$

Therefore, we have the following results:

Velocity profiles play an important role in many industrial processes, for example, removal of pollutants from plant discharge streams by absorptions and stripping of gases from waste water entirely depend on the relative rate of velocity profiles distribution. The velocity profiles across the boundary layer are shown in Fig.3.1 for various values of the variable permeability parameter $\mathrm{K}_{0}$ with respect to $t$ and $\mathrm{n}$. It can be clearly seen that $\mathrm{K}_{0}$ increases the velocity profile across the boundary layer decreases, when the permeability of the porous medium increases the resistance due to the porous medium decreases. In Figs (2-4) the velocity profiles are plotted for different values of the chemical reaction parameter $\gamma$, the heat source parameter $\mathrm{S}$ and the Dufour constant $\mathrm{D}_{u}$. Fig.3.2 elucidates that the velocity profile notably decreases with the higher values of the chemical reaction parameter. Fig.3.3 depicts that as the value of $\mathrm{S}$ increases, the velocity decreases. This happens because the influence of the heat source parameter reduces the fluid velocity across the boundary layer. The variation of velocity distribution across the boundary layer for several values of $\mathrm{D}_{u}$ is shown in Fig. 3.4. It can be observed that as $\mathrm{D}_{u}$ increase this leads to an increase in velocity at the vicinity of the plate. It is found that an increase in $\mathrm{D}_{u}$ causes a rise in fluid velocity, which suggests that the diffusion-thermo effect accelerated the fluid. The effects of the thermal radiation parameter $\mathrm{F}$ and the magnetic field parameter on velocity profiles are represented in Fig. 3.5 and Fig. 3.6. Fig.3.5 indicates that velocity increases with an 
increase in the radiation parameter. A magnetic field may be expected to stabilize a flow against the transition to turbulent flow. The cases of the magnetic field in the direction of flow, and normal to the flow, have been studied, and the stabilizing effect observed. From Fig 3.6, it is obvious that the velocity decreases when the magnetic field increases. This is because the applied transverse magnetic field will result in a resistive type of force i.e. the Lorentz force which tends to resist the fluid flow thus reducing the velocity.

The temperature profile for various values of $\mathrm{F}, \mathrm{S}, \mathrm{D}_{u}$ are described in the figures 3.7, 3.8 and 3.9. Fig.3.7 shows that an increase in the radiation parameter decrease the heat transfer in the thermal boundary layer because the higher values of radiation parameter increases the absorption coefficient. It is known that an increase in the heat source parameter has a tendency to diminish heat transfer, which results in a decrease in temperature as shown in Fig. 3.8. It can be clearly seen from Fig. 3.9 that the diffusive thermal effects slightly affect the fluid temperature. It suggests the variation of the thermal boundary-layer with the Dufour number ( $\mathrm{Du}$ ). It can be noticed that the thermal boundary layer thickness decreases with an increase in the Dufour number. The fluid temperature decreases for higher values of $\mathrm{D}_{u}$ Fig.1 3.10, 3.11 accounts for the influence of $\gamma$ and Sc, respectively, on the concentration distribution. It can be seen from Fig. 3.10 that concentration notably decreases for higher values of the chemical reaction parameter, which indicates that the diffusion rates can be changed by the chemical reaction parameter. The Schmidt number (Sc) embodies the ratio of the momentum diffusivity to the mass (species) diffusivity. It physically relates the relative thickness of the hydrodynamic boundary layer and mass-transfer (concentration) boundary layer. Figure 11 shows how concentration decreases as Sc increases. Fig. 3.12 shows the effect of the Dufour number $\mathrm{D}_{u}$ on skin friction $\tau$. It increases as $\mathrm{D}_{u}$ increases. Fig. 3.13 illustrates the variation of the surface heat transfer $\mathrm{N}_{u}$ with the suction velocity parameter A for different values of the thermal radiation parameter $\mathrm{F}$. It is clear that $\mathrm{N}_{u}$ decreases with the increasing values of F. Fig. 3.14 shows the rate of mass transfer for different values of the chemical reaction parameter $\gamma$. It is noticeable that with an increase of $\gamma$, the rate of mass transfer decreases. Fig 3.15 shows the effect on velocity when the chemical reaction parameter and the slip flow parameter have not been taken into account. The results are similar to Helmy [8].

The translational and angular velocity profile for different values of $\mathrm{D}_{u}, \mathrm{~F}, \alpha, \mathrm{K}_{0}$ for a polar fluid are described here. Fig. 3.16 and Fig. 3.17 show the translational velocity profile and angular velocity profile for different values of the Dufour effect parameter $\mathrm{D}_{u}$. It can be seen that translational velocity decreases as Du increases while the reverse effect is observed for the angular velocity profile. In Fig. 3.18 and 3.19 the velocity is plotted against different values of the thermal radiation parameter $\mathrm{F}$ and variability parameter $\mathrm{K}_{0}$. The figures indicate that with the increasing $\mathrm{F}$ and decreasing $\mathrm{K}_{0}$ the translational velocity increases. An increase in the porosity parameter leads to enhanced velocity profiles because it reduces the drag force. On the other side, the angular velocity profile decreases when both $\mathrm{K}_{0}$ and $\mathrm{F}$ increase. It is due to the fact that the momentum boundary layer thickness decreases when the thermal radiation parameter increases. The effect of the viscosity ratio $\alpha$ on the translational velocity and the microrotation profiles across the boundary layer are presented in Fig. 3.20 and 3.21. It can be seen that as the viscosity ratio $\alpha$ increases the translational and microrotation velocity increases. Figs. 3.22 and 3.23 illustrate the variation in translational velocity and angular velocity distribution for different values of the 


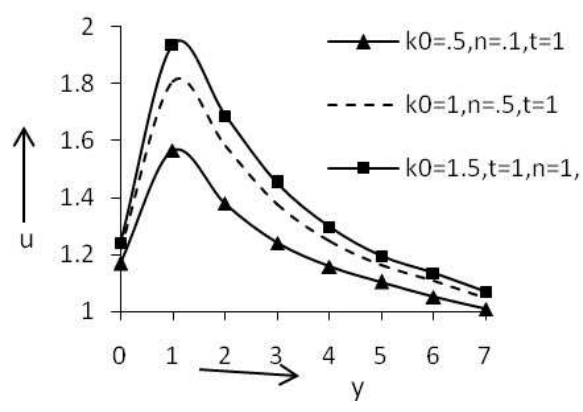

Fig. 3.1: Effect of $\mathrm{K}_{0}, \mathrm{n}, \mathrm{t}$ on Velocity profile

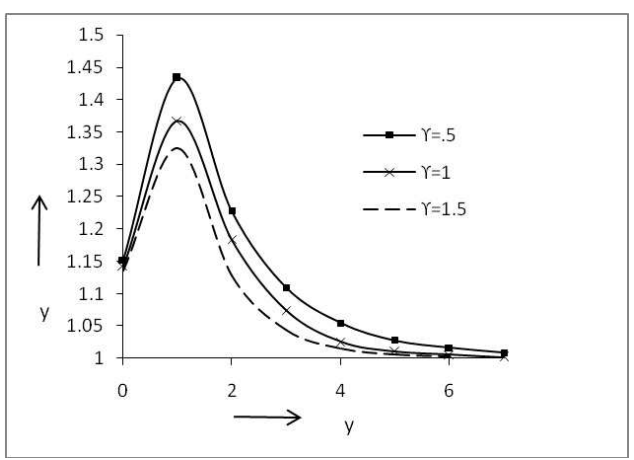

FIG. 3.2: Effect of $\gamma$ on Velocity profile

slip flow parameter $\mathrm{h}$. It is clear from the figure that an increase in the slip flow parameter leads to enhanced velocity. Figs. 3.24, 3.25 are plotted to show the influence of the Dufour number $\mathrm{D}_{u}$, the radiation parameter $\mathrm{F}$, on the temperature distribution, respectively. It can be noticed that an increase in the Dufour number increases the heat transfer, which is shown in Fig. 3.24. The effect of the thermal radiation parameter F is displayed in Fig. 3.25 It shows that an increase in radiation results in diminished heat transfer to the thermal boundary layer. Fig. 3.26 illustrates the variation of the surface heat transfer $\mathrm{N}_{u}$ with the suction velocity parameter A for different values of the thermal radiation parameter $\mathrm{F}$. It can be seen that $\mathrm{N}_{u}$ increases with the increasing values of F. Fig. 3.27 shows the rate of mass transfer for different values of the chemical reaction parameter $\gamma$. It is noticeable that when the values of $\gamma$ increase, the rate of mass transfer declines. 


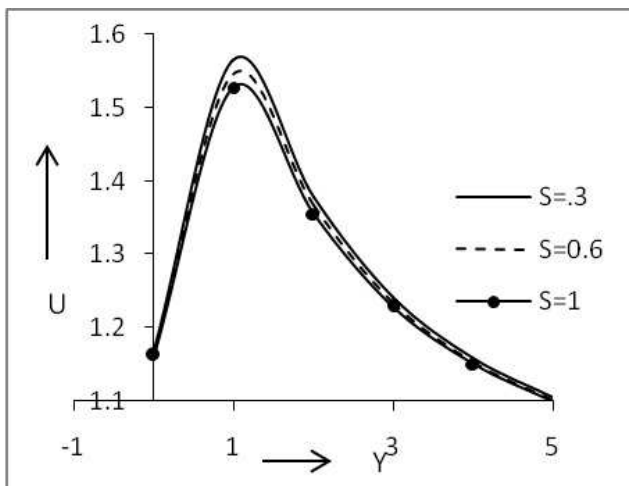

FIG. 3.3: Effect of S on Velocity profile

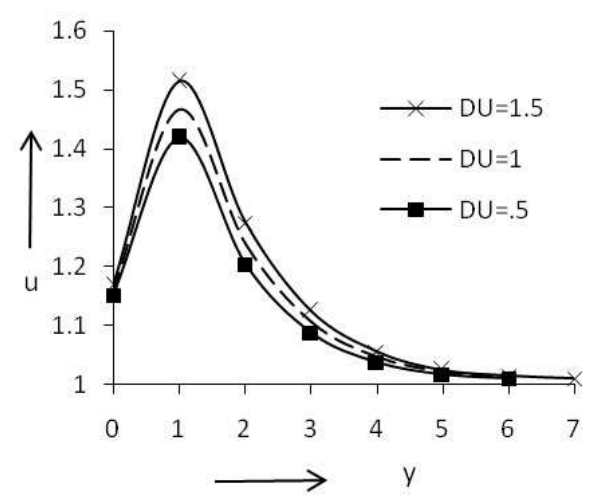

FIG. 3.4: Effect of $D_{u}$ on Velocity profile 


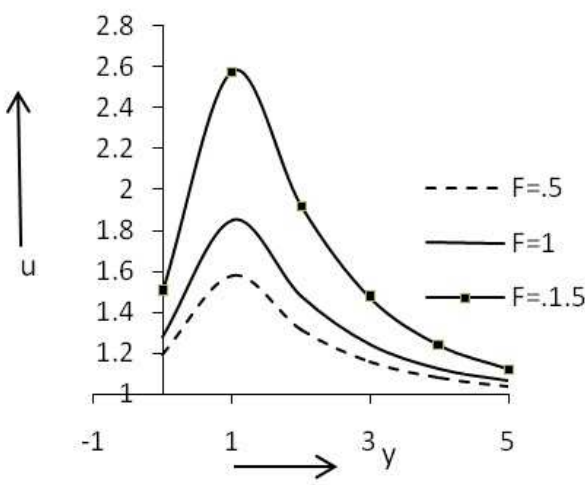

FIG. 3.5: Effect of F on Velocity profile

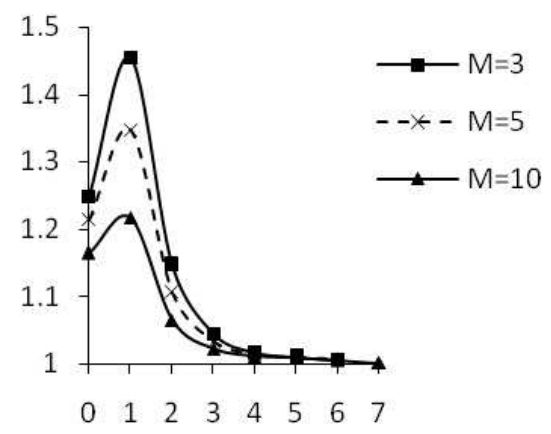

FIG. 3.6: Effect of $M$ on Velocity profile

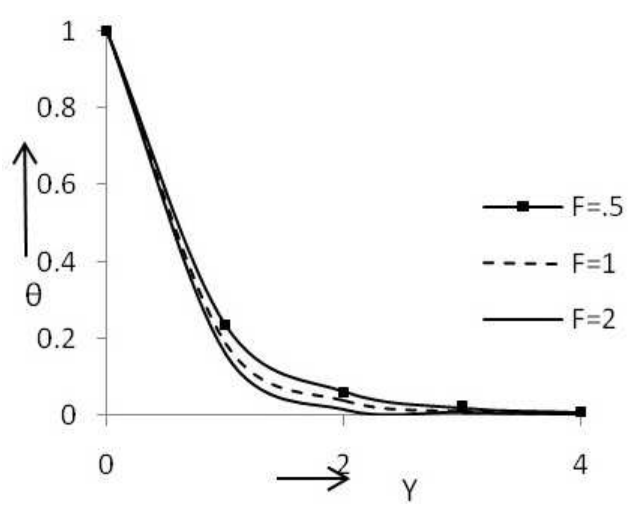

FIG. 3.7: Effect of $F$ on temperature profile 


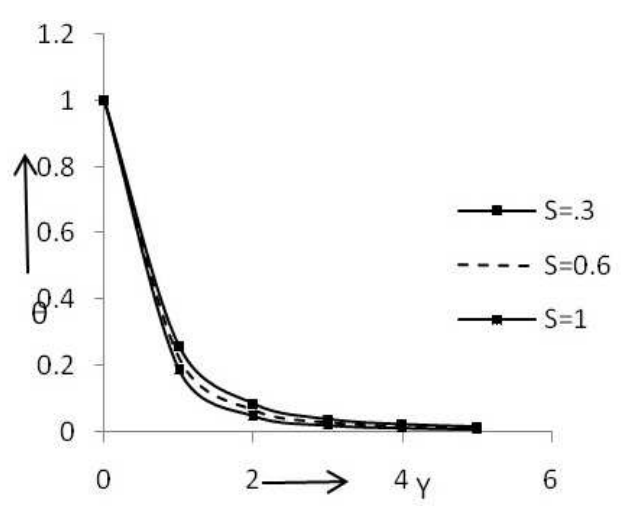

FIG. 3.8: Effect of $S$ on temperature profile

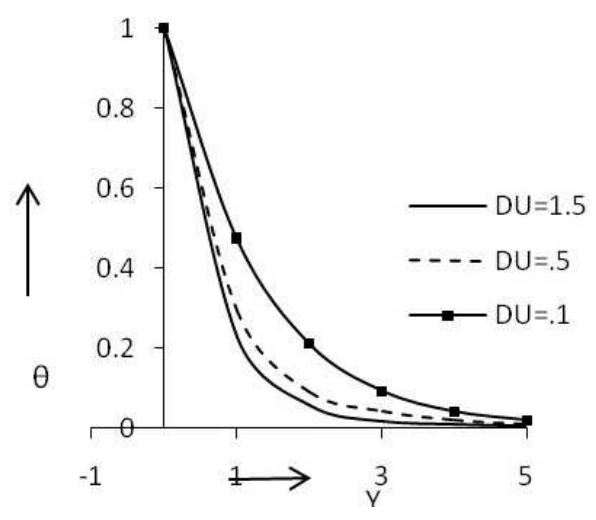

FIG. 3.9: Effect of $D_{u}$ on temperature profile

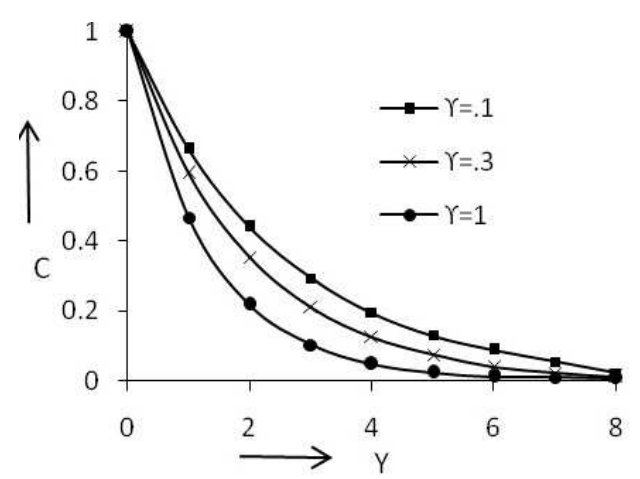

FIG. 3.10: Effect of $\gamma$ on Concentration profile 


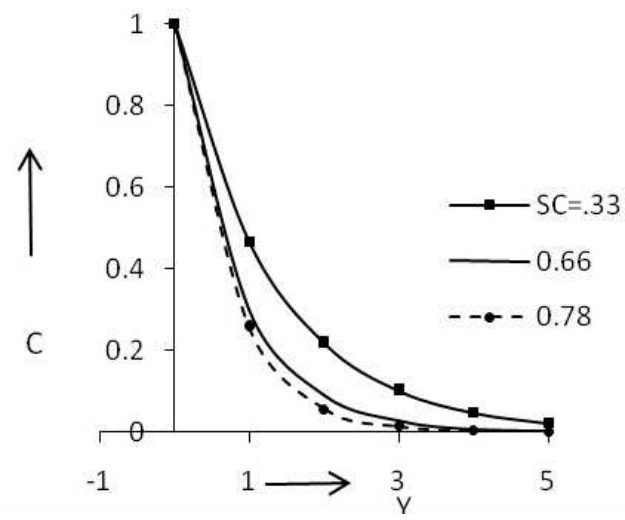

FIG. 3.11: Effect of $S_{c}$ on Concentration profile

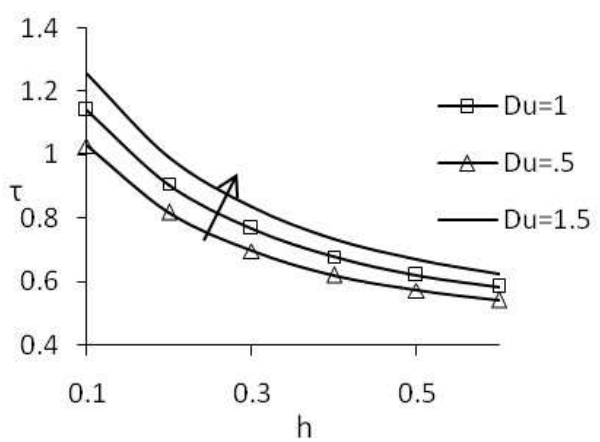

FIG. 3.12: Effect of $D_{u}$ on skin friction

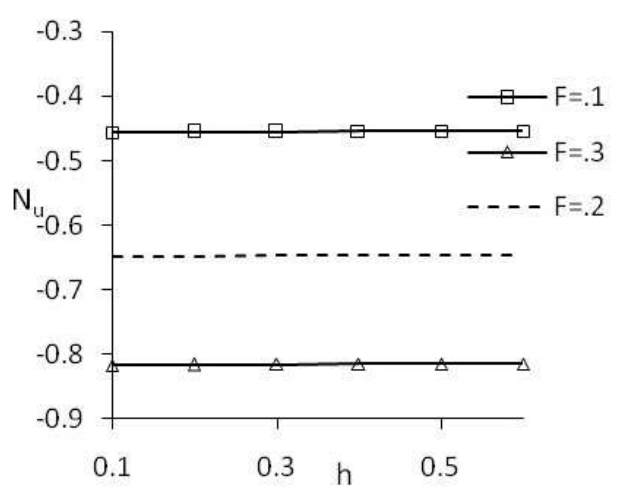

Fig. 3.13: Effect of $F$ on Nusselt number 


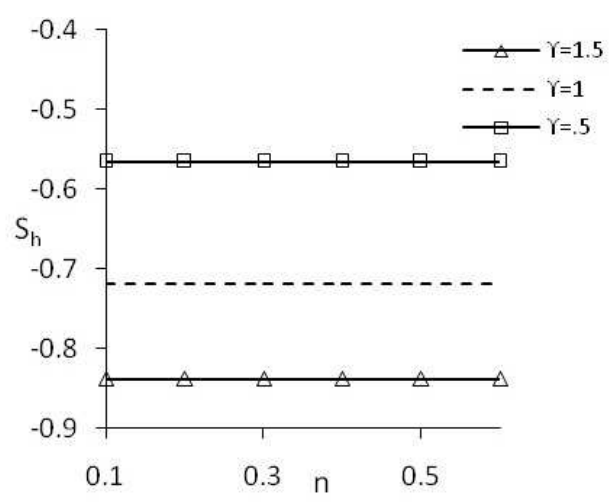

FIG. 3.14: Effect of $\gamma$ on Sharewood number

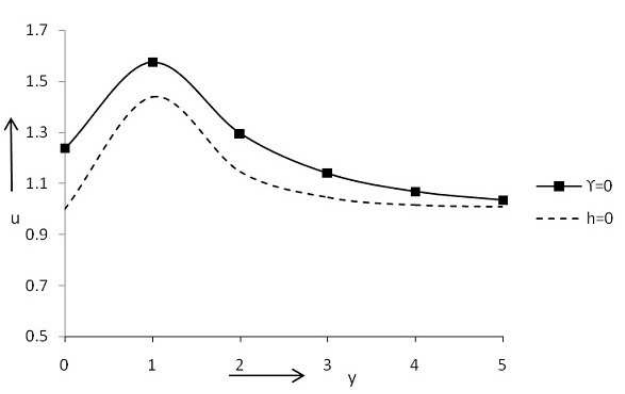

FIG. 3.15: Effect on velocity when $\gamma=0, h=0$

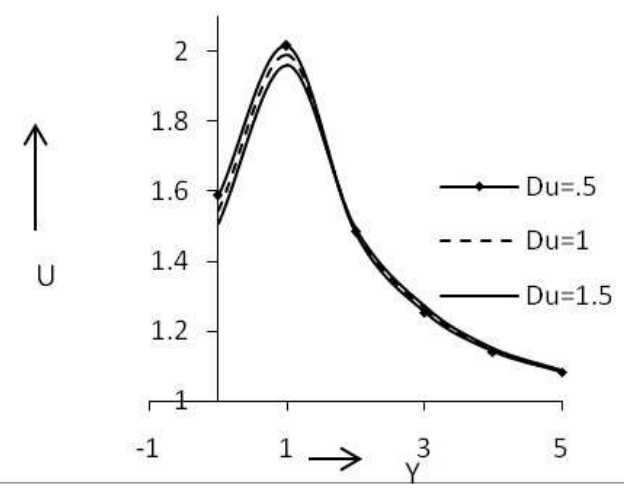

FIG. 3.16: Effect of $D_{u}$ on translational velocity profile 


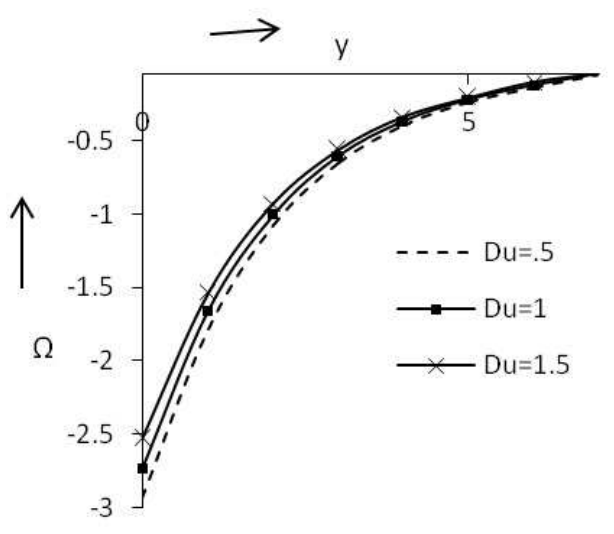

FIG. 3.17: Effect of $D_{u}$ on angular velocity profile

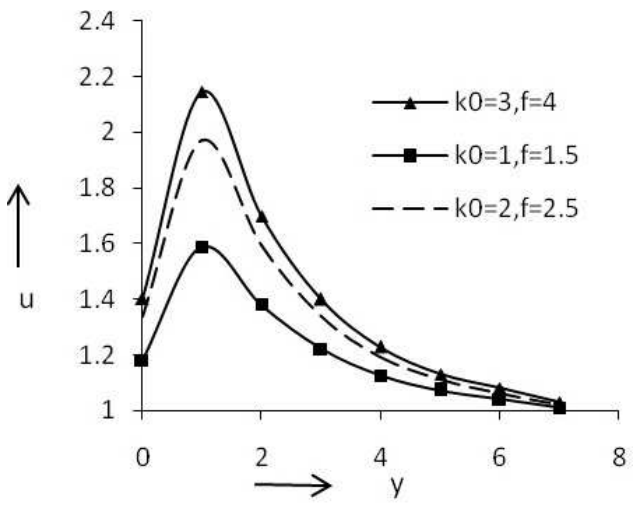

FIG. 3.18: Effect of $K_{0}$ and $F$ on translational velocity profile 


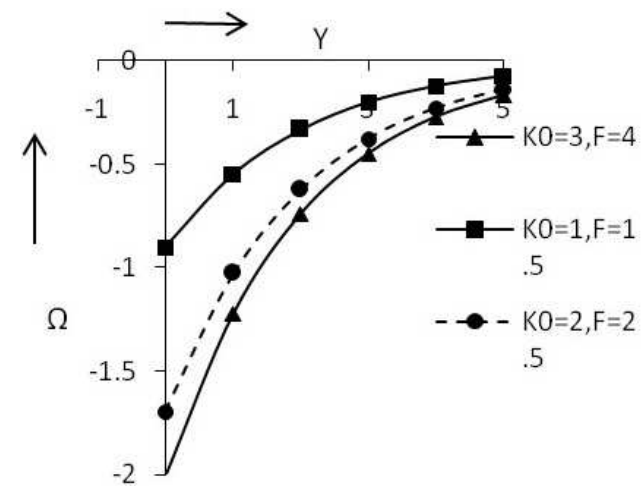

FIG. 3.19: Effect of $K_{0}$ and $F$ on angular velocity profile

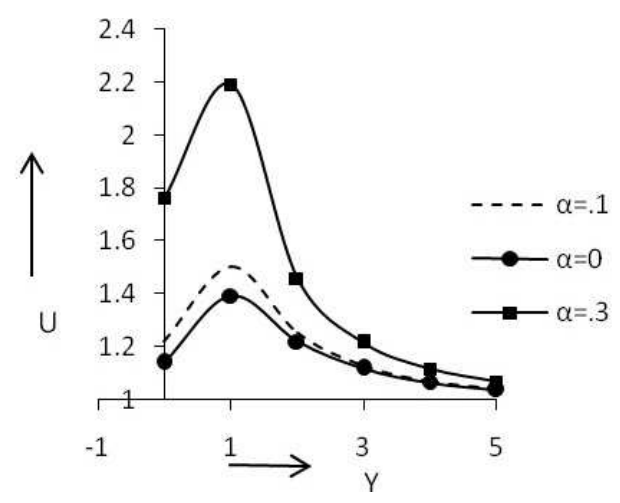

Fig. 3.20: Effect of $\alpha$ on translational velocity profile

All the parameters used in the paper are given below:

\begin{tabular}{|l|l|}
\hline Parameter & Values \\
\hline Thermal Grashof number $\left(G_{r}\right)$ & 5 \\
\hline Solutal Grashof number $\left(G_{c}\right)$ & 5 \\
\hline variable permeability $\left(K_{0}\right)$ & .5 \\
\hline Chemical reaction parameter $(\gamma)$ & .5 \\
\hline Heat source parameter $(\mathrm{S})$ & 1 \\
\hline Dufour effect parameter $\left(D_{u}\right)$ & .5 \\
\hline Radiation parameter $(\mathrm{F})$ & 1 \\
\hline Hartmann number $(\mathrm{M})$ & 5 \\
\hline Schmidt number $\left(\mathrm{S}_{c}\right)$ & .33 \\
\hline $\mathrm{n}=1$, Prandtl number $\left(\mathrm{P}_{r}\right)$ & .71 \\
\hline
\end{tabular}




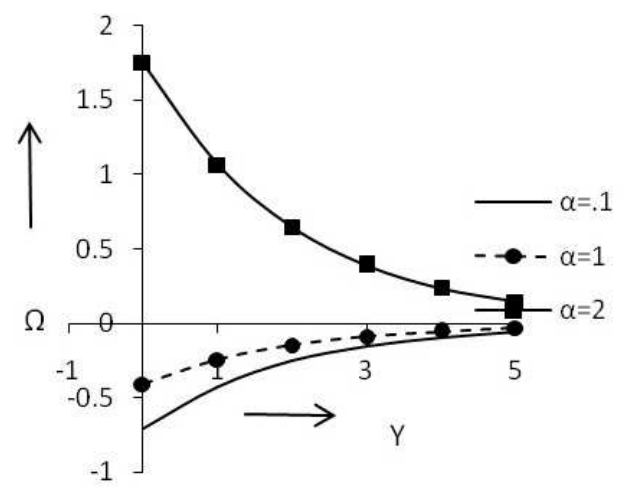

FIG. 3.21: Effect of $\alpha$ on angular velocity profile

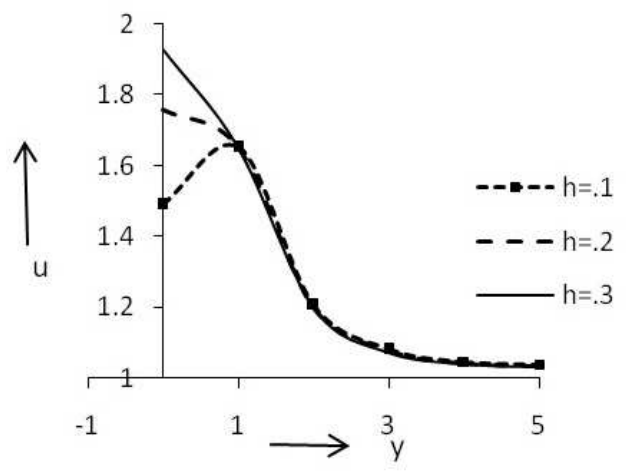

FIG. 3.22: Effect of $h$ on translational velocity profile

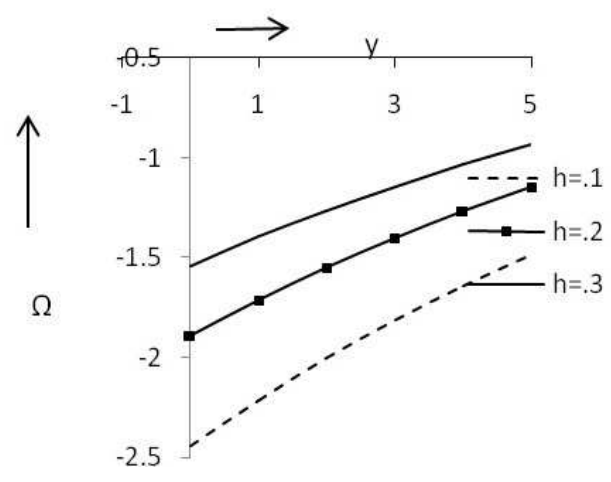

FIG. 3.23: Effect of $h$ on angular velocity profile 


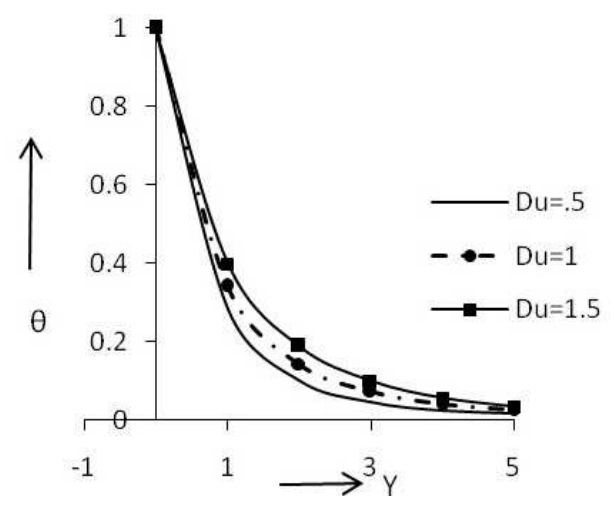

FIG. 3.24: Effect of $D_{u}$ on Temperature profile

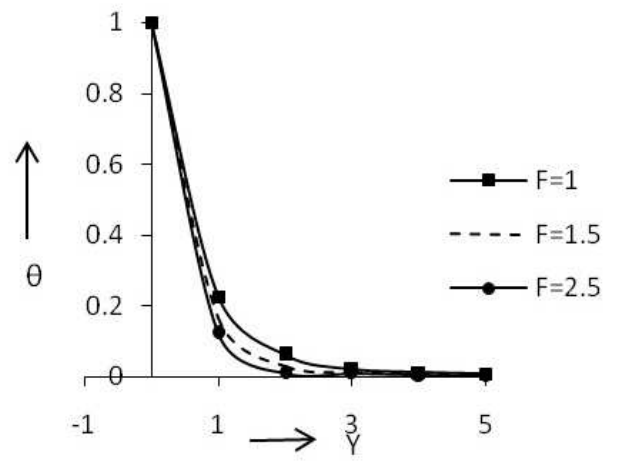

Fig. 3.25: Effect of $F$ on Temperature profile

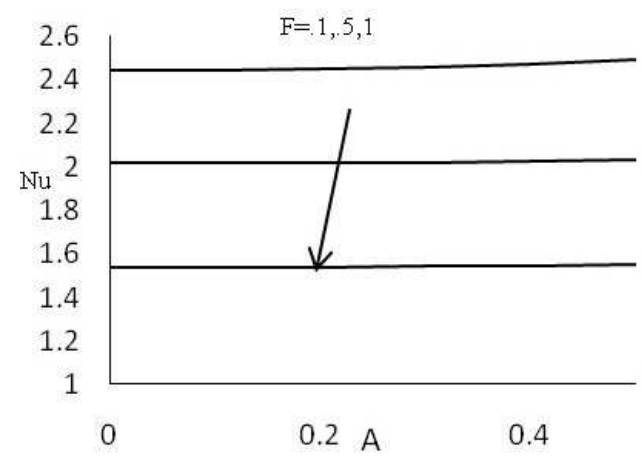

FIG. 3.26: Rate of heat transfer for various values of $F$ 


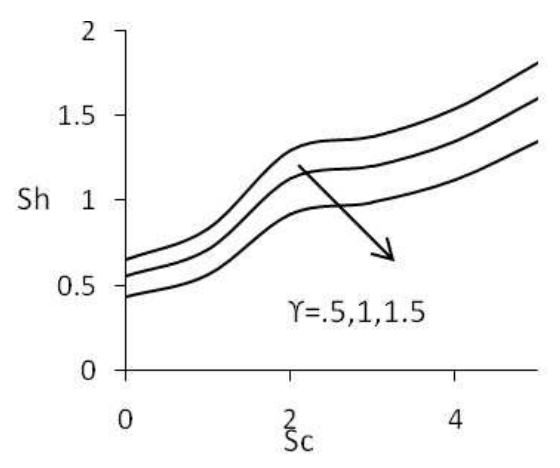

FIG. 3.27: Rate of Mass transfer for various values of $\gamma$

\section{Conclusion}

The aim of this paper is to study the unsteady MHD heat and mass transfer of the free convection flow of an incompressible, viscous, electrically conducting Newtonian and polar fluid past a semi-infinite porous plate embedded in a porous medium subjected to the presence of a transverse magnetic field with a diffusion-thermo and heat source. The method can be applied to small perturbation approximation. Important characteristics of the fluid flow, as well as heat and mass transfer, have been obtained and presented

The following conclusions are set out:

1. The velocity of a fluid increases with increasing values of the permeability parameter $K$ both in air and water.

2. The velocity for both a Newtonian and polar fluid increases as $D_{u}$ increases.

3. The velocity increases with decreasing values of the chemical reaction parameter $\Upsilon$.

4. The fluid temperature decreases with increasing values of $F$ and $S$.

5. Skin friction increases as $\mathrm{D}_{u}$ increases.

6. The translational velocity profiles increase with increasing values of the slip flow parameter (h), while the reverse effect is observed for micro-rotation velocity.

7. The Nusselt number and the Sharewood number decrease with decreasing values of F and $\gamma$, respectively.

Some constants have not been included for the sake of brevity of the paper.

\section{Acknowledgments}

The authors express their sincere thanks to the referees for their valuable suggestions and comments. 


\section{RE F E R E N C ES}

1. Ede, A.J., "Advances in free convection, advances in heat transfer." Academic Press, New York, 1967, Vol.4.

2. Martynenko, O.G., Berzovsky, A.A. and Sokovishin, A.Y. "Laminar free convection from a vertical plate.” Int. J. Heat Mass Transfer. 1984, Vol. 27 (6), P. 869.

3. Lee, S. and Yovanovich, M.M., "Linearization of natural convection from vertical plate with arbitrary heat flux distribution." J. Heat Transfer, 1992 , 114, p. 909.

4. Pantokraters, A., "Laminar free convection in water with variable physical properties adjacent to vertical plate with uniform heat flux", Int. J. Heat Mass Transfer, 2003, 46, p.725.

5. Vafai, K. (Ed.), "Handbook of porous media". Talor E Francis, Baton Roca, FL. 2005

6. Nield, D.A. and Bejan A., "Convection in porous media." Third ed., Springer, New York, 2006.

7. Cheng, P. and Minkowycz, W.J., "Free convection about a vertical flat plate embedded in porous medium with application to heat transfer from a dike." J.Geophys. Res . 1977, Vol.82 (14), p. 2040-2044.

8. Helmy, K.A., "MHD Unsteady Free Convection Flow past a Vertical Porous Plate." ZAMM, 1998, Vol.78 (4), p. 255-270.

9. Kim, Y.J., "Unsteady MHD convective heat transfer past a semi-infinite vertical porous moving plate with variable suction.” Int. J. Eng. Sci., 2000, Vol.38 (8), p. 833-845.

10. Das, S.S., Sahoo, S.K. and Dash, G.C., "Numerical solution of mass transfer effects on unsteady flow past an accelerated vertical porous plate with suction." Bull. Malays. Math. Sci. Soc., 2006, Vol. 29 (1), p. 33-42.

11. Eringen, A.C., "Simple microfluids.” Int. J. Energy Sci., 1964, Vol.2, p. 205-217.

12. Eringen, A.C., "Theory of micropolar fluids." J. Math. Mech., 1966, Vol.16, p.1-18.

13. Eringen, A.C., "Theory of Thermomicrofluids." J. Math. Anal. Appl., 1972, Vol. 38, p. 480-496.

14. Soundalgekar,V.M., "Unsteady free convection flow of an elastico-viscous fluid past an infinite vertical porous plate." J. Chem. Eng. Sci., 1971, Vol. 26, p. 2043-2050.

15. Rees, D.A.S. and Pop, I., "Free convection boundary layer flow of a micropolar fluid from a vertical flat plate", IMA J. Appl. Math.61, 1998, 179-197.

16. El-Amin, M. F., "Magnetohydrodynamic free convection and mass transfer flow in micropolar fluid with constant suction.” J. Magn.Magn. Mater, 2001, Vol. 234, p. 567-574.

17. Kim, Y. J., "Heat and Mass transfer in MHD micropolar flow over a vertical moving plate in a porous medium." Trans Porous Media, 2004, Vol. 56, p. 17-37.

18. Uddin, Z. , Kumar, Manoj and Harmand, S.," influence of thermal radiation and heat generation//absorption on MHD heat transfer flow of a micropolar fluid past a wedge with hall and ion slip currents" J. of Thermal Science. 2014, Vol.18 (2), p.489-502.

19. Umamaheswar, M. et al.: Numerical investigation of MHD free convection flow of a nonNewtonian fluid past an impulsively started vertical plate in the presence of thermal diffusion and radiation absorption. J. Alexandria Engineering Journal., 2016, Vol. 55 (3), p. 2005-2014.

20. Adrian, P., "Influence of a magnetic field on heat transfer by natural convection from vertical surfaces in porous media considering Soret and Dufour effects." Int. J. Heat Mass Transfer, 2004, Vol. 47, p. 1476-1472.

21. Mahdy, A., "MHD non-Darcian free convection from a vertical wavy surface embedded in porous media in the presence of Soret and Dufour effect", Int. Commun. Heat Mass Transfer, 2009, Vol. 36 (10), p.1067-1074. 
22. Mahdy, A., "Soret and Dufour effect on double diffusion mixed convection from a vertical surface in a porous medium saturated with a non-Newtonian fluid." J. Non-Newtonian Fluid Mechanics., 2010, Vol.165, p. 568-575.

23. Srinivasacharya, D., and Ram Reddy, Ch., "Effects of Soret and Dufour on mixed convection heat and mass transfer in a micropolar fluid with heat and mass fluxes." Int. J. Appl. Math. Mech., 2010, Vol. 6 (21), p. 8-21.

24. Pal, D., and Mondal, H., "MHD non Darcian mixed convection heat and mass transfer over non linear stretching sheet with Soret-Dufour effect and chemical suction." Int. Commun. Heat Mass Transfer, 2011, Vol. 38, p. 463-467.

25. Pal, D., Mondal, H., "Effect of Soret-Dofour chemical reaction and thermal radiation on MHD non-darcy unsteady mixed convective heat and mass transfer through over a stretching sheet." Comm.Nonlinear Sci.Numer.Simulation., 2011, Vol. 6, p. 1942-1958.

26. Hossain, M.M. and Khatun, M., "Study of diffusion-Thermo Effect on laminar mixed convection flow and heat transfer from a vertical surface with induced magnetic field." Int. J. Appl. Math. Mech., 2012, Vol. 8 (5), p. 40-60.

27. A.J. Omowaye, A.I. Fagbade, A.O. Ajayi :Dufour and soret effects on steady MHD convective flow of a fluid in a porous medium with temperature dependent viscosity: Journal of the Nigerian Mathematical Society ., 2015 ,Vol.34, p.343-360.

28. Srinivasa Raju R. : "Effects of Soret and Dufour on natural convective fluid flow past a vertical plate embedded in porous medium in presence of thermal radiation via FEM.” . J of KSIAM., 2016, Vol.20 (4), P.309-332.

29. Sharma, P. K., and Chaudhary, R.C., "Effect of variable suction on transient free convective viscous incompressible flow past a vertical plate in a slip-flow regime." Emirates Journal of Engineering Research., 2003, Vol. 8 (2), p. 33-38.

30. Khandelwal, K., Gupta, A., Poonam, Jain N.C., "The effects of couple stress on the flow through a porous medium with variable permeability in slip flow regime." Ganita, 2003, Vol. 54 (2), p. 203-212.

31. Sharma, P.K., "The influence of periodic temperature and concentration on unsteady free convective viscous incompressible flow and heat transfer past a vertical plate in a slip flow." Matemáticas: Enseñanza Universitaria ., 2005, Vol.13, p. 51-62.

32. Muthuraj, R., and Srinivas, S., "Influence of magnetic field and wall slip conditions on steady flow between parallel flat wall and a long wavy wall with Soret effect." J. Nav. Architect. Mar. Eng., 2009, Vol. 6, p. 62-71.

33. Ellahi R., Shivanian, E., Abbasbandy, S., Rahman, S.U., and Hayat, T.: "Analysis of steady flows in viscous fluid with heat/mass transfers and slips effects". Int. J. Heat Mass Transfer, 2012, Vol. 55, p. 6384-6390.

34. Sreenivasulu P. , Poornima T. and Bhaskar Reddy N. : "Thermal radiation effect on MHD boundary layer slip flow past a permeable exponential stretching sheet in the presence of joule heating and viscous dissipation". Journal of Applied Fluid Mechanics, 2016, Vol. 9 (1), p. 267-278.

35. Kayalvizhi , M. , Kalaivanan, R. , Vishnu Ganesh , N. and Ganga B.: "Velocity slip effects on heat and mass fluxes of MHD viscous-Ohmic dissipative flow over a stretching sheet with thermal radiation”, Ain Shams Engineering Journal., 2016, Vol.7, p.791-797..

36. Kandasamy, R., Periasamy, K., and Prabhu, K.K.S., "Chemical reaction, heat and mass transfer on MHD flow over a vertical stretching surface with heat source and thermal stratification effects." Int. J. Heat Mass Transfer, 2005, Vol. 48 (21-22), p. 4557-4561. 
37. Chaudhary, R.C., Jha, A.K., "Effects of chemical reaction on MHD micropolar fluid flow past a vertical plate in slip flow regime." J. Appl. Math. Mech., 2006, Vol. 29 (9), p. 1-16.

38. Patil, P.M., and Kulkarni, P.S., "Effect of chemical reaction on free convective flow of a polar fluid through porous medium in the presence of thermal heat generation." Int. J. Therm. Sci., 2008, Vol. 47, p. 1043-1054.

39. Pal, D. and Talukdar, B., "Buoyancy and chemical reaction effects on MHD mixed convection heat and mass transfer in porous medium with thermal radiation and ohmic heating." Comm. Nonlinear Sci. Nume. Simulation., 2010, Vol. 15 (10), p. 2878-2893.

40. Pal, D. and Mondal, H., "MHD non-Darcian mixed convection heat and mass transfer over a non-linear stretching sheet with Sore-Dufour effects and chemical reaction." Int. Commun. Heat Mass Transfer., 2011, Vol. 38 (4), p. 463-467.

41. Surya Narayana Reddy, K., Sreedhar, M. Babu, S. Vijaya Kumar Varma and Bhaskar Reddy, N., "Hall Current And Dufour Effects On Mhd Flow Of A Micropolar Fluid Past A Vertical Plate In The Presence Of Radiation Absorption And Chemical Reaction" IOSR Journal of Mathematics,2014, Vol. 10 A (204),p.106-121.

42. Jonnadula Manjula, et al.:" Influence of Thermal Radiation and Chemical Reaction on MHD Flow, Heat and Mass Transfer over a Stretching Surface". Procedia Engineering, 2015, Vol.127, P.1315-1322.

43. Rawat, S., Kapoor, S. and Bhargava, R. : "MHD Flow Heat and Mass Transfer of Micropolar Fluid over a Nonlinear Stretching Sheet with Variable Micro Inertia Density, Heat Flux and Chemical Reaction in a Non-darcy Porous Medium”. J. of Appl. Fluid Mech., 2016, Vol. 9 (1), P. 321-331.

44. Murthy, S.N., Feyen, J., "Influence of variable permeability on the dispersion of a chemically reacting solute in porous media.” Int. J. Eng. Sci., Vol., 1989, 27 (12), p. 1661-1671.

45. Hassanien, I.A., and Obied Allah, M.H., "Oscillatory hydromagnetic flow through a porous medium with variable permeability in the presence of free convection and mass transfer flow." Int. Cотmu. Heat Mass Transfer, 2002, Vol. 29, p. 567-575.

46. Mahmoud, M.A.A., "Thermal radiation effects on MHD flow of a micropolar fluid over a stretching surface with variable thermal conductivity", Physica A 375, 2007, p. 401-410.

47. Ibrahim, F.S., Elaiw, A.M., Bakr, A.A., "Influence of viscous dissipation and radiation on unsteady MHD mixed convection flow of micropolar fluids." Appl. Math. Inform. Sci., 2008, Vol. 2, p. 143-162.

48. Makinde, O.D., and Moitsheki, R.J., "On non-perturbative techniques for thermal radiation effect on natural convection past a vertical plate embedded in a saturated porous medium." Math. Prob. Eng., 2008, 689074 (11p.).

49. Das, K., "Effect of chemical reaction and thermal radiation on heat and mass transfer flow of MHD micropolar fluid in a rotating frame of reference." Int. J. Heat Mass Transfer, 2011, Vol. 54 , p. 3505-3513.

50. Sreenivasulu P., Poornima T. and Bhaskar N. Reddy .: Thermal Radiation Effects on MHD Boundary Layer Slip Flow Past a permeable Exponential Stretching Sheet in the Presence of Joule Heating and Viscous Dissipation. Journal of Applied Fluid Mechanics.,2016, Vol. 9 (1), p. 267-278,

51. Dyke V.M, “perturbation method in fluid mechanics.” parabolic press, 1995

52. Rees, D.A.S. and Bossom, A.P., "The Blasisun boundary layer flow of micropolar fluid," Int. Eng. Sci., 1996, Vol. 34, p. 113. 


\section{Komal Choudhary}

Department of Mathematics,

Department of Mathematics and Informatics

Laxmidevi Institute of Engineering and Technology, Chikani,

Alwar-301 001, India

dr. kchoudhary@yahoo.com

Abhay Kumar Jha

Department of Mathematics,

JECRC University, Jaipur-302 022, India

itsabhay@rediffmail.com

Lakshmi Narayan Mishra

Department of Mathematics,

Lovely Professional University, Jalandhar-Delhi, G.T. Road,

Phagwara, Punjab 144 411, India

L. 1627 Awadh Puri Colony Beniganj,

Phase -III, Opposite - Industrial Training Institute (I.T.I.),

Ayodhya Main Road, Faizabad-224 001, Uttar Pradesh, India

lakshminarayanmishra04@gmail.com

Vandana

Department of Management Studies,

Indian Institute of Technology, Madras,

Tamil Nadu-600 036, India

vdrai1988@gmail.com 